\title{
Avaliação dos Fatores de Prevalência Sobre o Estado Nutricional de Crianças em Idade Escolar
}

\author{
Kerulyn Maria Chanivski Machado ${ }^{1}$, Juliana de Lara Castagnoli², \\ Mayra Lopes de Oliveira ${ }^{3}$, Flávia Teixeira ${ }^{4}$, Jaqueline Machado Soares ${ }^{5}$, Daiana Novello ${ }^{6}$
}

\begin{abstract}
RESUMO
Objetivo: Avaliar os fatores de prevalência sobre o estado nutricional de crianças em idade escolar. Método: Participaram da pesquisa 626 crianças de ambos os sexos, com idade entre 7 a 10 anos, matriculadas em 18 escolas municipais. Foram avaliadas as variáveis sexo, idade e escolaridade. $O$ estado nutricional foi avaliado pelo Índice de Massa Corporal (IMC). Resultados: A média de idade, peso e altura foram de $8,49 \pm 0,79$ anos, $33,58 \pm 9,32 \mathrm{~kg}$ e $1,34 \pm 0,07$ metros, respectivamente. A maioria das crianças frequentava o $4^{\circ}$ ano do Ensino Fundamental, com estado nutricional de eutrofia $(58,31 \%)$, entretanto um grande número de alunos apresentou excesso de peso $(40,73 \%)$. Não houve prevalência significativa $(p>0,05)$ do estado nutricional sobre as variáveis de sexo, idade e escolaridade. Apesar disso, uma maior frequência de obesidade foi observada em indivíduos do sexo masculino e com idade entre 9 e 10 anos e que frequentavam o $4^{\circ}$ ano do Ensino Fundamental. Conclusão: 0 estado nutricional de crianças em idade escolar não é influenciado pelo sexo, idade e escolaridade.
\end{abstract}

Palavras-chave: Infância. Escola. Obesidade.

\section{EVALUATION OF PREVALENCE FACTORS ON THE NUTRITIONAL STATE OF CHILD AGE CHILDREN}

\section{ABSTRACT}

Objective: Evaluate the prevalence factors on the nutritional status of school-age children. Method: The study included 626 children of both sexes, aged 7 to 10 years, enrolled in 18 municipal schools. They were evaluated as variables of sex, age and education. Nutritional status was assessed by Body Mass Index (BMI). Results: The mean age, weight and height were 8.49 \pm 0.79 years, $33.58 \pm 9.32 \mathrm{~kg}$ and $1.34 \pm 0.07$ meters, respectively. Most children attend the $4^{\text {th }}$ grade of elementary school, with nutritional status of eutrophic (58.31\%), but a large number of overweight students (40.73\%). There was no significant difference $(p>0.05)$ in nutritional status on the variables sex, age and education. Nevertheless, a higher frequency of obesity was observed in males aged between 9 and 10 years and attending the $4^{\text {th }}$ grade of elementary school. Conclusion: The nutritional status of school-age children is not influenced by sex, age and education.

Keywords: Childhood. School. Obesity.

\footnotetext{
Nutricionista pelo Centro Universitário Campo Real. http://lattes.cnpq.br/8828045853300635. https://orcid.org/0000-0002-3932-1958. kerulyn.maria1@ outlook.com

${ }^{2}$ Mestranda pelo Programa de Pós-Graduação Interdisciplinar em Desenvolvimento Comunitário pela Universidade Estadual do Centro-Oeste (Unicentro). http://lattes.cnpq.br/6091846848961295. https://orcid.org/0000-0003-3476-508X. julara2008@hotmail.com

${ }^{3}$ Mestranda pelo Programa de Pós-Graduação Interdisciplinar em Desenvolvimento Comunitário pela Universidade Estadual do Centro-Oeste (Unicentro). http://lattes.cnpq.br/0355769248014879. https://orcid.org/0000-0001-8502-317X. mayra.lopes2010@gmail.com

${ }^{4}$ Mestre pelo Programa de Pós-Graduação Interdisciplinar em Desenvolvimento Comunitário pela Universidade Estadual do Centro-Oeste (Unicentro). http:// lattes.cnpq.br/7183567082019128. https://orcid.org/0000-0002-9615-827X. teixeiraflavia19@gmail.com

${ }^{5}$ Mestre pelo Programa de Pós-Graduação Interdisciplinar em Desenvolvimento Comunitário pela Universidade Estadual do Centro-Oeste (Unicentro). http:// lattes.cnpq.br/6152550809902240.https://orcid.org/0000-0001-6990-7725. jaquue.s@gmail.com

${ }^{6}$ Doutora em Tecnologia dos Alimentos pela Universidade Estadual de Campinas (Unicamp). Docente do curso de Nutrição e do Programa de Pós-Graduação Interdisciplinar em Desenvolvimento Comunitário da Universidade Estadual do Centro-Oeste (Unicentro). http://lattes.cnpq.br/1756064975510727. https:// orcid.org/0000-0003-0762-5292.nutridai@gmail.com
} 


\section{INTRODUÇÃO}

No Brasil, a prevalência de obesidade vem crescendo de forma alarmante entre adultos e crianças. Dados recentes mostraram que mais da metade da população adulta do país encontra-se com excesso de peso (54\%), acometendo $57,3 \%$ dos homens e $51,2 \%$ das mulheres. Além do mais, foi registrada uma elevada frequência de obesidade $(18,9 \%)$ entre indivíduos adultos (BRASIL, 2018). A maior porcentagem de sobrepeso e obesidade ocorre nas regiões Sul $(53,8 \%$ e $17,4 \%$, respectivamente), Sudeste ( $49 \%$ e $14,7 \%$, respectivamente) e Centro-Oeste ( $48,6 \%$ e $15,1 \%$, respectivamente) do país. As mesmas regiões brasileiras são responsáveis pelos elevados índices médios de sobrepeso $(37,2 \%)$ e de obesidade $(16,6 \%)$ entre crianças (IBGE, 2010). Medidas governamentais mundiais vêm sendo adotadas contribuindo para o controle e o combate dos casos de sobrepeso e obesidade, especialmente em crianças (WHO, 2018). No Brasil, exemplos dessas ações são a Política Nacional de Alimentação e Nutrição-PNAN (BRASIL, 2012a), o Programa Nacional de Alimentação Escolar-PNAE (BRASIL, 2018b), o Programa Saúde da Escola-PSE (BRASIL, 2015), a regulamentação da publicidade de alimentos (BRASIL, 2010) e as Leis Municipais e Estaduais da cantina saudável (BRASIL, 2007). Essas ações conjuntas buscam melhorar a qualidade de vida, e propõem garantir a oferta de alimentos seguros e saudáveis no ambiente escolar, além de promover e proteger a saúde das crianças.

A infância é considerada uma fase relevante para a realização de ações que busquem a promoção da saúde e a prevenção da obesidade infantil. O sobrepeso e a obesidade na infância são causados principalmente pela ingestão calórica excessiva e o baixo consumo de frutas, hortaliças, fibras e carboidratos complexos (IFPRI, 2014). Além disso, observa-se um aumento do uso de aparelhos eletrônicos e declínio na prática de atividade física (LÓPEZ-BARRÓN; JIMÉNEZ; BACARDÍ, 2015). O nível socioeconômico da família da criança também pode interferir no estado nutricional, uma vez que influencia na aquisição de alimentos e no padrão de atividade física (FREDERICK; SNELLMAN; PUTNAM, 2014). Outros fatores, como o estado civil dos pais, idade da mãe, tempo de licença maternidade e acompanhamento pré-natal, têm uma relação direta com a introdução precoce de alimentos ultraprocessados na infância, o que pode contribuir para o excesso de peso (LONGO-SILVA et al., 2017).

Pesquisas já demonstraram que crianças com sobrepeso e obesidade apresentam maiores chances para o desenvolvimento de patologias crônicas futuras, como diabetes mellitus, doença renal (BASSAIN VAL-
DÉS et al., 2015), hipertensão arterial, dislipidemias (BRAY; BOUCHARD, 2014), dentre outras. O excesso de peso também pode desencadear problemas psicológicos e psiquiátricos, influenciando de forma negativa no estado emocional. Frequentemente, crianças com desvios nutricionais são rejeitadas na escola e nos grupos de amigos, sendo excluídas de atividades rotineiras, como brincadeiras e práticas de atividade física. Com isso, são comuns os problemas com imagem corporal, quadros de ansiedade, estresse, transtornos de humor e depressão (RODRIGUEZ-AYLLON et al., 2018), além de sentimentos de tristeza e de inferioridade. Todos esses efeitos podem perdurar na fase adulta, provocando, muitas vezes, doenças ainda mais graves.

Uma ferramenta que pode auxiliar na monitoração da saúde das crianças é a avaliação nutricional, posto que avalia o grau de risco nutricional, colaborando para instaurar medidas adequadas de saúde pública de prevenção e combate à obesidade (MUSSOI, 2014). Para a avaliação da composição corporal utiliza-se, em geral, o Índice de Massa Corporal (IMC), que é calculado dividindo-se o peso $(\mathrm{kg})$ pela altura ao quadrado $\left(\mathrm{m}^{2}\right)$ (WHO, 2017). Essa técnica apresenta diversas vantagens, como o baixo custo, a praticidade e a simplicidade na aferição das medidas. Sua objetividade e possibilidade de comparação com padrões de referência auxiliam principalmente em estudos populacionais (MUSSOI, 2014). Nesse contexto, o objetivo desta pesquisa foi avaliar os fatores de prevalência sobre o estado nutricional de crianças em idade escolar.

\section{MATERIAIS E MÉTODOS}

\section{População e Amostra}

O estudo caracteriza-se como epidemiológico e transversal, ocorrendo no período entre junho a agosto de 2018. Foi avaliada uma amostra representativa do total de crianças (9.600) em idade escolar (7-10 anos), matriculadas entre o 20 e o 50 ano em 36 escolas públicas municipais existentes na área urbana de Guarapuava, PR, Brasil. A determinação da amostra foi realizada em dois estágios: 1) as escolas foram selecionadas por meio de amostragem não probabilística por conveniência, sendo escolhida aquela do bairro com maior quantidade de alunos, totalizando-se, dessa forma, 18 escolas; 2) após a seleção das escolas, as crianças foram escolhidas por meio de amostragem aleatória simples, levando-se em consideração os seguintes parâmetros: número total de alunos matriculados do 20 ao 5 응 ano de escolas urbanas da cidade, nível de confiança de $95 \%$ e erro máximo aceito de $5 \%$, totalizando uma amostra representativa mínima de 368 alunos. 


\section{Avaliação do Estado Nutricional}

O peso $(\mathrm{kg})$ foi obtido em uma balança digital portátil (Tanita $\left.{ }^{\circ}, \mathrm{EUA}\right)$, com precisão de $100 \mathrm{~g}$, e a estatura $(\mathrm{m})$ foi verificada por meio de uma fita métrica inelástica $(100 \mathrm{~cm}$, precisão de $0,1 \mathrm{~cm})$ fixada à parede sem rodapé. Para o cálculo do IMC utilizou-se a seguinte fórmula: peso/estatura² (BRASIL, 2011). Os resultados foram expressos em valor de escore $z$ em relação à mediana da população de referência, por meio das Curvas de Crescimento para a idade de 5 a 19 anos preconizadas pela OMS (BRASIL, 2006/2007). O diagnóstico do estado nutricional foi avaliado considerando as crianças em conjunto e separadas por sexo, conforme a seguinte classificação: "magreza acentuada" (< escore-z - 3); "magreza" ( $\geq$ escore-z - 3 e < escore-z - 2); "eutrofia" (> escore- $z-2$ e $\leq$ escore- $z+1)$; "sobrepeso" (> escore- $z+1$ e $\leq$ escore- $z+2$ ); "obesidade" (> escore- $z+$ 2 e $\leq$ escore- $z+3$ ); "obesidade grave" (> escore- $z+3$ ). Para fins de comparação estatística, o estado nutricional também foi categorizado como descrito a seguir: a) "baixo peso" (magreza e magreza acentuada), quando escore-z < - 2; b) "eutrofia" (estado nutricional adequado), quando escore-z entre - $2 \mathrm{e}+1 ; \mathrm{c}$ ) "excesso de peso" (sobrepeso, obesidade e obesidade grave) quando escore-z > + 1 (BRASIL, 2006/2007). A categoria "eutrofia" foi adotada como grupo de referência.

\section{Análise Estatística}

A tabulação dos dados foi realizada no programa Epi Data (Data Management and Basic Statistical Analysis System, versão 3.1, Odense, Dinamarca). A normalidade dos dados foi verificada pelo teste Kolmogorov-Smirnov. Para a realização das análises, utilizou-se o software R empregando-se procedimentos de estatística descritiva e inferencial. Foi utilizado o teste Qui-Quadrado de Pearson ou exato de Fisher na comparação da prevalência dos indicadores em razão das variáveis independentes. Todos os testes foram analisados com um nível de $5 \%$ de significância $(p \leq 0,05)$.

\section{Questões Éticas}

O projeto foi aprovado pelo Comitê de Ética em Pesquisa Envolvendo Seres Humanos (Comep) da Unicentro, sob parecer no 3.089.447/2018. Ressalta-se que todos os preceitos éticos de pesquisa com seres humanos foram adotados, conforme recomenda a Resolução no 466/2012 do Conselho Nacional de Saúde (CNS) (BRASIL, 2012b). Os critérios de inclusão para a participação foram: crianças em idade escolar, regularmente matriculadas entre o 20 e 050 ano das escolas municipais urbanas de Guarapuava, PR e assinatura do Termo de Consentimento Livre e Esclarecido (TCLE) pelos responsáveis e do Termo de Assentimento (TA) pelo escolar. Os critérios de exclusão foram: idade abaixo ou acima do estabelecido, crianças não matriculadas nas escolas e nas séries selecionadas e não apresentar o TCLE ou TA assinados.

\section{RESULTADOS E DISCUSSÃO}

Participaram da pesquisa 626 crianças, 70,1\% acima da amostra mínima de 368 indivíduos. A média de idade foi de 8,49 anos, sem predominância entre os sexos. As médias de peso $(33,58 \pm 9,32 \mathrm{~kg})$ e de altura $(1,34 \pm 0,07 \mathrm{~m})$ encontram-se acima das recomendações para a idade e sexo nesses parâmetros, que é de $26,8 \mathrm{~kg} / 1,30 \mathrm{~m}$ para as meninas e $27 \mathrm{~kg} / 1,30$ $\mathrm{m}$ para os meninos (WHO, 2007). O IMC médio das crianças foi de $18,30 \mathrm{~kg} / \mathrm{m}^{2}$ (sobrepeso), enquanto a maior parte delas frequentava o $4^{\circ}$ ano do Ensino Fundamental. Apesar de a maioria das crianças estarem eutróficas, um grande número (255) foi classificado com o peso acima do ideal $(40,73 \%)$ para idade e sexo (WHO, 2007). Esses resultados corroboram outras pesquisas realizadas em países como Estados Unidos (ROUSE et al., 2019), China (YAN et al., 2018) e Argentina (CUESTA et al., 2018). No Brasil, casos similares foram encontrados em diferentes Estados, como Rio Grande do Sul (FRIEDRICH; ANTUNES; SCHUCH, 2016), Paraíba (PEDRAZA et al., 2017), São Paulo (MODOTTI; RODRIGUES; LUDWIG, 2017) e Rio de Janeiro (TEIXEIRA et al., 2017). Na Tabela 1 estão descritas as características gerais das crianças em idade escolar.

Tabela 1 - Características gerais das crianças em idade escolar. Guarapuava, PR

\begin{tabular}{lcccc}
\hline Variável & $\mathbf{n}$ & $\%$ & Média & $\begin{array}{c}\text { Desvio } \\
\text { padrão }\end{array}$ \\
\hline $\begin{array}{l}\text { Dados gerais } \\
\text { Idade (anos) }\end{array}$ & - & - & 8,49 & 0,79 \\
$\quad$ Peso (kg) & - & - & 33,58 & 9,32 \\
$\quad$ Altura (m) & - & - & 1,34 & 0,07 \\
$\quad$ Índice de Massa & - & - & 18,30 & 3,77 \\
$\quad$ Corporal (kg/m $\left.{ }^{2}\right)$ & & & & \\
Sexo & & & & - \\
Masculino & 313 & 50 & - & - \\
$\quad$ Feminino & 313 & 50 & - & - \\
Idade (anos) & & & & - \\
7 anos & 62 & 9,90 & - & - \\
8 anos & 249 & 39,78 & - & - \\
9 anos & 260 & 41,53 & - & - \\
10 anos & 55 & 8,79 & - & - \\
Escolaridade & & & & - \\
$3^{\circ}$ ano & 168 & 26,84 & - & - \\
$4^{\circ}$ ano & 446 & 71,25 & - & - \\
$5^{\circ}$ ano & 12 & 1,92 & - & - \\
Estado Nutricional & & & & - \\
Desnutrição & 6 & 0,96 & - & - \\
Eutrofia & 365 & 58,31 & - & - \\
Sobrepeso & 138 & 22,05 & - & - \\
Obesidade & 80 & 12,78 & - & - \\
Obesidade grave & 37 & 5,90 & - & - \\
\hline
\end{tabular}

N: 626.

Fonte: Os autores. 
Nos últimos anos, muitos países passaram por mudanças expressivas nos hábitos alimentares da população, que caracterizam as transições demográfica e epidemiológica. Esse processo foi marcado pela diminuição dos casos de desnutrição e aumento de sobrepeso e de obesidade em todas as faixas etárias (PEDERSOLI et al., 2015). Nesse sentido, já foi observada uma redução drástica no consumo de frutas, hortaliças e leite, especialmente pelas crianças, com o aumento da ingestão de alimentos industrializados, como bolachas, salgadinhos, refrigerantes e doces (VITOLO, 2014). Além do aumento da obesidade infantil, o baixo consumo de frutas e hortaliças pode desencadear carências nutricionais, o que interfere no crescimento e desenvolvimento (DUMKE; SCHALEMBERGUER; BENEDETTI, 2015). No Brasil, as carências nutricionais mais observadas são causadas pela deficiência de ferro, zinco e vitamina A (CARVALHO et al., 2015). Outro aspecto que contribui para o aumento do sobrepeso é a prática de atividade física insuficiente, especialmente entre crianças residentes em áreas urbanas (LIMBERGER et al., 2018).

Dentre os anos de 2000 a 2010 houve um aumento substancial na utilização de equipamentos eletrônicos, como videogame, computador e televisão nas residências (LIMBERGER et al., 2018). Esse fato colaborou para a redução da atividade física ao ar livre e, consequentemente, para o aumento do excesso de peso (MELO; SILVA; SANTOS, 2018). Existem outras situações que podem contribuir para o aumento dos casos de obesidade na infância. A mídia, por exemplo, investe cada vez mais em propagandas que apresentam influência direta na preferência pelo consumo de produtos industrializados (SILVA et al., 2015). Além disso, fatores, como baixo/alto peso ao nascer, genética, interrupção precoce do aleitamento materno, introdução inadequada da alimentação complementar, dentre outros, também podem contribuir para o excesso de peso na infância (WEFFORT; LAMOUNIER, 2017). A alimentação da criança está intimamente relacionada aos costumes familiares (MAIA; SETTE, 2015), uma vez que tais costumes determinam o tipo de alimento consumido. De forma similar, a escola pode influenciar na alimentação infantil, posto que existe a convivência com outros indivíduos que podem intervir no tipo de alimento ingerido e na prática de exercícios físicos (STEEVES et al., 2016).

Programas de educação alimentar e nutricional, quando realizados de forma conjunta e em ambientes globais, como a escola, auxiliam de forma positiva na formação de hábitos saudáveis duradouros (JONGENELIS et al., 2017). Exemplos de intervenções interdisciplinares são as hortas escolares (SILVA et al., 2014), as oficinas culinárias (REZENDE; NEGRI, 2015) e as oficinas musicais (SANTOS; BERGOLD, 2018). Sendo assim, a escola pode ser considerada um ambiente favorável e adequado para o desenvolvimento de ações educativas e formação de novos hábitos alimentares (VITOLO, 2014), com o intuito de prevenir e/ou reduzir efetivamente o excesso de peso na infância. Na Tabela 2 está descrita a prevalência das variáveis estratificadas conforme o estado nutricional (IMC/I) das crianças em idade escolar.

Não houve associação significativa $(p>0,05)$ entre o estado nutricional e as variáveis sexo, idade e escolaridade, como demonstrado por Dias et al. (2017). Apesar disso, é possível que a obesidade infantil seja mais frequente em crianças do sexo masculino (ZADKA; GO-

Tabela 2 - Prevalência e razão de chances (odds ratio bruto, multinomial) das variáveis estratificadas conforme o estado nutricional (IMC/I). Guarapuava, PR

\begin{tabular}{|c|c|c|c|c|c|c|c|c|c|c|c|}
\hline \multirow[t]{2}{*}{ Variáveis } & \multicolumn{2}{|c|}{ Baixo peso } & \multicolumn{2}{|c|}{ Eutrofia } & \multicolumn{2}{|c|}{ Excesso de peso* } & \multirow[t]{2}{*}{$p$} & \multirow{2}{*}{$\begin{array}{l}\text { Baixo peso } \\
\text { OR (IC 95\%) }\end{array}$} & \multirow[t]{2}{*}{$p$} & \multirow{2}{*}{$\begin{array}{c}\text { Excesso de peso* } \\
\text { OR (IC 95\%) }\end{array}$} & \multirow[t]{2}{*}{$p$} \\
\hline & $n$ & $\%$ & $\mathrm{n}$ & $\%$ & $\mathrm{n}$ & $\%$ & & & & & \\
\hline \multicolumn{12}{|l|}{ Sexo } \\
\hline Masculino & 2 & 33,3 & 175 & 47,9 & 136 & 53,3 & $0,299^{a}$ & $0,50(0,09-2,73)$ & $0,343^{b}$ & $1,25(0,91-1,72)$ & $0,167^{\mathrm{a}}$ \\
\hline Feminino & 4 & 66,7 & 190 & 52,1 & 119 & 46,7 & & 1 & & 1 & \\
\hline \multicolumn{12}{|l|}{ Idade (anos) } \\
\hline 7 a 8 anos & 5 & 83,3 & 181 & 49,6 & 125 & 49,0 & $0,251^{\mathrm{a}}$ & $5,13(0,60-44,17)$ & $0,105^{b}$ & $0,96(0,70-1,32)$ & $0,784^{a}$ \\
\hline 9 a 10 anos & 1 & 16,7 & 184 & 50,4 & 130 & 51,0 & & 1 & & 1 & \\
\hline \multicolumn{12}{|l|}{ Escolaridade } \\
\hline $3^{\circ}$ ano & 3 & 50,0 & 97 & 26,6 & 68 & 26,7 & $0,732^{\mathrm{a}}$ & $2,76(0,55-13,80)$ & $0,199^{b}$ & $0,99(0,69-1,41)$ & $0,936^{a}$ \\
\hline $4^{\circ}$ ano & 3 & 50,0 & 260 & 71,2 & 183 & 71,8 & & 1 & & 1 & \\
\hline $5^{\circ}$ ano & 0 & 0,0 & 8 & 2,2 & 4 & 1,6 & & $1,01(1,00-1,02)$ & $0,890^{b}$ & $0,72(0,21-2,43)$ & $0,416^{b}$ \\
\hline
\end{tabular}

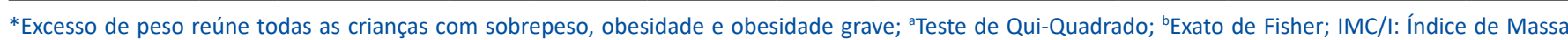
Corporal para Idade; N: 626. 
ZDZIK; HUSZCZ, 2019); isso porque podem apresentar maior preferência por alimentos com maiores teores de açúcar e calorias, o que acaba contribuindo para a obesidade (JALKANEN et al., 2017). Já as meninas consomem mais frutas, hortaliças e sopas (MORENO-BLACK; STOCKARD, 2018), em especial porque têm uma cobrança familiar e social maior por um corpo magro.

A fase de repleção pré-puberal, que acontece por volta dos 8 aos 10 anos de idade, é marcada pelo acúmulo de tecido adiposo corporal, como forma de reserva energética para o estirão de crescimento e desenvolvimento que ocorrerá na adolescência (VITOLO, 2014). Assim, já foi demonstrado que crianças com idade entre 9 e 10 anos apresentam maior prevalência de sobrepeso ou obesidade em relação às faixas etárias inferiores (SILVA et al., 2016). Não existe, porém, um consenso na literatura que descreva essa faixa etária como preditora de excesso de peso (ANJOS; SILVEIRA, 2017). O excesso de peso entre os escolares pode ter um efeito negativo sobre a cognição mental, causando um declínio no desempenho escolar (KHAN et al., 2015). Estudos revelam, contudo, que elevados teores de nutrientes, como o ferro, por exemplo, podem ajudar no funcionamento adequado do cérebro, na memória e na aprendizagem (IBRAHIM et al., 2017). Por isso, ações multi e interdisciplinares são necessárias para verificar os determinantes dos agravos nutricionais, e também identificar as intervenções mais adequadas para a promoção da saúde e controle do sobrepeso/obesidade nessa fase da vida.

\section{CONCLUSÃO}

A maioria das crianças em idade escolar, residentes em Guarapuava, PR, Brasil, apresenta eutrofia, contudo há uma elevada frequência de excesso de peso. Variáveis como sexo, idade e escolaridade não apresentam prevalência significativa sobre o estado nutricional das crianças. Apesar disso, uma maior frequência de obesidade foi observada em indivíduos do sexo masculino, com idade entre 9 e 10 anos, e que frequentavam o $4^{\circ}$ ano do Ensino Fundamental. Dessa forma, sugere-se o monitoramento contínuo do perfil nutricional de crianças na fase escolar, além do desenvolvimento de ações interdisciplinares educativas, voltadas à promoção de hábitos saudáveis e à prevenção da obesidade.

\section{AGRADECIMENTOS}

Os autores agradecem ao Fundo Paraná/Seti pela concessão dos recursos financeiros e ao Programa Universidade sem Fronteiras, referentes ao edital n. 07/2017/Seti, Paraná, Brasil.

\section{REFERÊNCIAS}

ANJOS, L. A.; SILVEIRA, W. D. B. Estado nutricional dos alunos da Rede Nacional de Ensino de Educação Infantil e Fundamental do Serviço Social do Comércio (Sesc), Brasil, 2012. Ciência \& Saúde Coletiva, v. 22, n. 5, p. 1.725-1.734, 2017.

BASSAIN VALDÉS, J. M. et al. Malnutrición por exceso e hipertensión arterial en niños y adolescentes de un área de salud. Revista Archivo Médico de Camagüey, v. 19, n. 3, p. 220-228, 2015.

BRASIL. Ministério da Saúde. Secretaria de Vigilância em Saúde. Departamento de Vigilância de Doenças e Agravos não Transmissíveis e Promoção da Saúde. Vigitel Brasil 2017 vigilância de fatores de risco e proteção para doenças crônicas por inquérito telefônico: estimativas sobre frequência e distribuição sociodemográfica de fatores de risco e proteção para doenças crônicas nas capitais dos 26 Estados Brasileiros e no Distrito Federal em 2017. Brasília: Ministério da Saúde, 2018a.

BRASIL. Ministério da Educação. Manual de apoio para atividades técnicas do nutricionista no âmbito do PNAE. Programa Nacional de Alimentação Escolar. Brasília: FNDE, 2018b.

BRASIL. Ministério da Saúde. Ministério da Educação. Caderno do Gestor do PSE - Programa Saúde na Escola. Brasília: Ministério da Saúde, 2015.

BRASIL. Ministério da Saúde. Secretaria de Atenção à Saúde. Departamento de Atenção Básica. Política Nacional de Alimentação e Nutrição (PNAN). Brasília: Ministério da Saúde, 2012a.

BRASIL. Resolução no 466, de 12 de dezembro de 2012. Aprova diretrizes e normas regulamentadoras de pesquisas envolvendo seres humanos. 2012b. Disponível em: https://conselho.saude.gov.br/resolucoes/2012/Reso466.pdf. Acesso em: 19 abr. 2020.

BRASIL. Ministério da Saúde. Secretaria de Atenção à Saúde. Departamento de Atenção Básica. Orientações para a coleta e análise de dados antropométricos em serviços de saúde: Norma Técnica do Sistema de Vigilância Alimentar e Nutricional - Sisvan. Brasília: Ministério da Saúde, 2011.

BRASIL. Agência Nacional de Vigilância Sanitária (Anvisa). Resolução RDC $n^{\circ} 24$, de 15 de junho de 2010. Dispõe sobre a oferta, propaganda, publicidade, informação e outras práticas correlatas, cujo objetivo seja a divulgação e a promoção comercial de alimentos considerados com quantidades elevadas de açúcar, de gordura saturada, de gordura trans, de sódio e de bebidas com baixo teor nutricional. Diário Oficial da União. Brasília, DF: Poder Executivo, 15 jun. 2010. BRASIL. Ministério da Saúde. Secretaria de Atenção à Saúde. Departamento de Atenção Básica. Coordenação-Geral da Política de Alimentação e Nutrição. Incorporação das curvas de crescimento da Organização Mundial da Saúde de 2006 e 2007 no SISVAN. 2006/2007. Disponível em: https://www.sprs.com.br/sprs2013/bancoimg/131209104419oms2006_2007. pdf. Acesso em: 19 Abr. 2020. 
BRASIL. Ministério da Saúde. Secretaria de Atenção à Saúde. Departamento de Atenção Básica. Coordenação Geral da Política de Alimentação e Nutrição. Regulamentação da Comercialização de Alimentos em Escolas no Brasil: experiências estaduais e municipais. Brasília: Ministério da Saúde, 2007.

BRAY, A. G.; BOUCHARD, C. Handbook of obesity. 4. ed. Boca Raton: Taylor \& Francis Group, 2014.

CARVALHO, C. A. et al. Consumo alimentar e adequação nutricional em crianças brasileiras: revisão sistemática. Revista Paulista de Pediatria, v. 33, n. 2, p. 211-221, 2015.

CUESTA, L. L. et al. Anthropometric and biochemical assessment of nutritional status and dietary intake in school children aged 6-14 years, Province of Buenos Aires, Argentina. Archivos Argentinos de Pediatria, v. 116, n. 1, p. 34-46, 2018.

DIAS, V. M. et al. Influência dos conhecimentos em nutrição e fatores socioeconômicos no excesso de peso infantil. Biosaúde, v. 19, n. 1, p. 26-38, 2017.

DUMKE, E.; SCHALEMBERGUER, J. T. S.; BENEDETTI, F. J. Consumo e análise de gorduras totais de alimentos ultraprocessados ingeridos por pré-escolares. Disciplinarum Scientia: Ciências da Saúde, v. 16, n. 1, p. 89-99, 2015.

FREDERICK, C. B.; SNELLMAN, K.; PUTNAM, R. D. Increasing socioeconomic disparities in adolescent obesity. Proceedings of the National Academy of Sciences of the United States of America, v. 111, n. 4, p. 1.338-1.342, 2014.

FRIEDRICH, R. R.; ANTUNES, T.; SCHUCH, I. Percepção materna do estado nutricional de crianças em escolas municipais de Porto Alegre - RS. Demetra: Alimentação, Nutrição \& Saúde, v. 11, n. 1, p. 211-223, 2016.

IBGE. Instituto Brasileiro de Geografia e Estatística. Pesquisa de Orçamentos Familiares (POF) 2008-2009: antropometria e estado nutricional de crianças, adolescentes e adultos no Brasil. Rio de Janeiro: IBGE, 2010.

IBRAHIM, A. et al. Iron therapy and anthropometry: A case-control study among iron deficient preschool children. Egyptian Pediatric Association Gazette, v. 65, n. 3, p. 95100, 2017.

IFPRI. International Food Policy Research Institute. Global nutrition report 2014: actions and accountability to advance nutrition and sustainable developmen. Washington: Ifpri, 2014.

JALKANEN, H. et al. Eating behaviour is associated with eating frequency and food consumption in 6-8 year old children: the Physical Activity and Nutrition in Children (PANIC) study. Appetite, v. 114, n. 1, p. 28-37, 2017.

JONGENELIS, M. I. et al. Assessing parents' receptiveness to a vegetable-focussed in-school nutrition intervention. $A p$ petite, v. 117, n. 1, p. 359-364, 2017.

KHAN, N. A. et al. Central adiposity is negatively associated with hippocampal-dependent relational memory among overweight and obese children. The Journal of Pediatrics, v. 166, n. 2, p. 302-308, 2015.
LIMBERgER, T. et al. Presença de obesidade em escolares: estudo comparativo entre a zona urbana e rural de Santa Cruz do Sul-RS. Revista Brasileira de Obesidade, Nutrição e Emagrecimento, v. 12, n. 76, p. 1.113-1.118, 2018.

LONGO-SILVA, G. et al. Age at introduction of ultra-processed food among preschool children attending day-care centers. Jornal de Pediatria, v. 93, n. 5, p. 508-516, 2017.

LÓPEZ-BARRÓN, R. G.; JIMÉNEZ, A. C.; BACARDÍ, M. G. Modifiable environmental obesity risk factors among elementary school children in a México-US Border city. Nutrición Hospitalaria, v. 31, n. 5, p. 2.047-2.053, 2015.

MAIA, C. S. C.; SETTE, R. S. Consumo alimentar infantil em uma cidade do sul de Minas: uma proposta de inspiração antropológica. Organizações Rurais \& Agroindustriais, v. 17, n. 1, p. 87-100, 2015.

MELO, K. S.; SILVA, K. L. G. D.; SANTOS, M. M. D. Avaliação do estado nutricional e consumo alimentar de pré-escolares e escolares residentes em Caetés-PE. Revista Brasileira de Obesidade, Nutrição e Emagrecimento, v. 12, n. 76, p. 1.039-1.049, 2018.

MODOTTI, S.; RODRIGUES, J. R.; LUDWIG, K. M. Comparação do estado nutricional de escolares matriculados na rede pública de ensino da periferia e do centro da cidade de Assis- SP. Journal of the Health Sciences Institute, v. 35, n. 3, p. 182-186, 2017.

MORENO-BLACK, G.; STOCKARD, J. Salad bar selection patterns of elementary school children. Appetite, v. 120, n. 1, p. 136-144, 2018.

MUSSOI, T. D. Avaliação nutricional na prática clínica: da gestação ao envelhecimento. São Paulo: Guanabara Koogan, 2014.

PEDERSOLI, A. G. A. et al. Avaliação do estado nutricional de crianças com idade entre 7 a 10 anos no município de Porto Velho - Rondônia. Revista Saber Científico, v. 4, n. 1, p. 19-27, 2015.

PEDRAZA, D. F. et al. Estado nutricional e hábitos alimentares de escolares de Campina Grande, Paraíba, Brasil. Ciência \& Saúde Coletiva, v. 22, n. 2, p. 469-477, 2017.

REZENDE, M. F.; NEGRI, S. T. Educação alimentar e nutricional associada a oficinas culinárias com alunos em uma escola pública. Revista Eletrônica de Extensão, v. 12, n. 20, p. 21-35, 2015.

RODRIGUEZ-AYLLON, M. et al. Physical fitness and psychological health in overweight/obese children: a cross-sectional study from the Active Brains project. Journal of Science and Medicine in Sport, v. 21, n. 2, p. 179-184, 2018.

ROUSE, H. et al. Prevalence, patterns, and predictors: a statewide longitudinal study of childhood obesity. Journal of School Health, v. 89, n. 4, p. 237-245, 2019.

SANTOS, A. P. M.; BERGOLD, L. B. Oficinas musicais: a utilização do lúdico e da música para Educação Alimentar e Nutricional com escolares. Rasbran - Revista da Associação Brasileira de Nutrição, v. 9, n. 2, p. 88-93, 2018.

SILVA, D. C. A. et al. Percepção de adolescentes sobre a prática de alimentação saudável. Ciência \& Saúde Coletiva, v. 20, n. 11, p. 3.299-3.308, 2015. 
SILVA, D. C. F. et al. Horta escolar: interdisciplinaridade, reflexão ambiental e mudanças de hábitos alimentares. Revista de Ciências Exatas Tecnologia, v. 9, n. 9, p. 47-55, 2014.

SILVA, K. E. S. et al. Nutritional status of schoolchildren aged 7-10 years enrolled in public and private schools of Cascavel, Paraná, Brazil. Revista de Nutrição, v. 29, n. 5, p. 699708, 2016.

STEEVES, E. T. A. et al. Social influences on eating and physical activity behaviours of urban, minority youths. Public Health Nutrition, v. 19, n. 18, p. 3.406-3.416, 2016.

TEIXEIRA, F. C. et al. Metabolic syndrome's risk factors and its association with nutritional status in schoolchildren. Preventive Medicine Reports, v. 6, n. 1, p. 27-32, 2017.

VITOLO, M. R. Nutrição - da gestação ao envelhecimento. 2. ed. Rio de Janeiro: Rubio, 2014.

WEFFORT, V. R. S.; LAMOUNIER, J. A. Nutrição em pediatria: da neonatologia a adolescência. 2. ed. São Paulo: Manole, 2017.

WHO. Word Health Organization. Taking action on childhood obesity. 2018. Disponível em: https://apps.who.int/ iris/bitstream/handle/10665/274792/WHO-NMH-PND-ECHO-18.1-eng.pdf?sequence=1\&isAllowed=y. Acesso em: 9 jan. 2020.

WHO. World Health Organization. Guideline: assessing and managing children at primary health-care facilities to prevent overweight and obesity in the context of the double burden of malnutrition. 2017. Disponível em: https://apps.who.int/iris/bitstream/handle/ 10665/259133/9789241550123-eng. pdf? sequence $=1$. Acesso em: 10 jan. 2020.

WHO. World Health Organization. Growth reference data for 5-19 years. 2007. Disponível em: https://www.who.int/ growthref/en/. Acesso em: 10 jan. 2020.

YAN, X. Y. et al. Trend in the nutritional status of children aged 2-7 years in Luoding city, China: a panel study from 2004 to 2013. Plos One, v. 13, n. 10, p. 1-12, 2018.

ZADKA, K.; GOZDZIK, E. P.; HUSZCZ, D. R. Relation between environmental factors and children's health behaviors contributing to the occurrence of diet-related diseases in central Poland. International Journal of Environmental Research and Public Health, v. 16, n. 1, p. 1-14, 2019. 